\title{
Percepción social de usuarias atendidas exclusivamente por enfermeras en la etapa perinatal ${ }^{1}$
}

\author{
Danelia Gómez Torres ${ }^{2}$; Gabriela Téllez Rojas ${ }^{3}$; Pedro Miguel Santos Dinis Pareira ${ }^{4}$; Aida Maris Peres ${ }^{5}$
}

Institución: Universidad Autónoma del Estado de México.

\section{RESUMEN}

El artículo muestra la atención exclusiva de enfermeras en una maternidad, cuyo objetivo fue analizar las representaciones sociales de las usuarias acerca de la atención brindada por las enfermeras obstetras en la institución. Es una investigación con enfoque cualitativo, descriptivo; direccionado por la teoría de las representaciones sociales de Moscovici. Se entrevistó a 34 mujeres atendidas en la maternidad. El análisis de datos se efectuó mediante marco interpretativo, seleccionando las ideas destacadas por medio de técnica cromática; en seguida, se discute los datos empíricos confrontados con la teoría. Las usuarias señalan que la atención recibida se inscribió en un marco de humanización, responsabilidad y confianza, lo cual posibilita la atención de un parto de acuerdo con sus costumbres que, con base en la teoría, refleja autonomía profesional. Se concluye que el modelo de atención muestra que el cuidado de enfermería se basa en conocimientos, experiencia y valores que potencializan la práctica, desde la que se aprecia el cuidado humano, cálido y personalizado.

Palabras clave: enfermería-perinatal; percepción-social; parto-humanizado; servicios-de-enfermería.

DOI: https://doi.org/10.15517/revenf.v0i35.33666

${ }^{1}$ Fecha de recepción: 01 de marzo 2018

2 Doctora en Enfermería. Docente e Investigadora de la Facultad de Enfermería y Obstetricia de la Universidad Autónoma del Estado de México. Toluca, México. Correo electrónico: gomezdanelia@usa.net

${ }^{3}$ Licenciada en Enfermería de la Facultad de Enfermería y Obstetricia de la Universidad Autónoma del Estado de México. Toluca,

México. Correo electrónico: Gabriela Téllez Rojas. Correo electrónico: gabrielatellezrojas@hotmail.com

${ }^{4}$ Doctor en Ciencias de la Salud. Profesor de la Escuela Superior de Enfermería de Coímbra, Portugal. Correo electrónico:

parreira@esenfc.pt

${ }^{5}$ Doctora en Enfermería. Profesora de la Universidad Federal do Paraná. Curitiba-Paraná-Brasil. Correo electrónico:

aidamaris.peres@gmail.com 


\title{
Social perception of users served exclusively by nurses at the perinatal stage ${ }^{1}$
}

\author{
Danelia Gómez Torres ${ }^{2}$; Gabriela Téllez Rojas ${ }^{3}$; Pedro Miguel Santos Dinis Pareira ${ }^{4}$ Aida Maris Peres ${ }^{5}$
}

Institution: Autonomous Mexico State University.

\begin{abstract}
The article shows the exclusive attention of nurses in a maternity, whose objective was to analyze the social representations of the users about the attention given by the obstetric nurses in the institution. It is a research with a qualitative, descriptive approach; Directed by the theory of social representations of Moscovici. 34 women attended in the maternity were interviewed. The data analysis was carried out through an interpretative framework, selecting the ideas highlighted by means of chromatic technique; next, the empirical data confronted with the theory is discussed. The users point out that the care received was part of a framework of humanization, responsibility, and trust, which makes it possible to attend a delivery according to their customs, which, based on theory, reflects professional autonomy. It is concluded that the care model shows that nursing care is based on knowledge, experience, and values that potentiate the practice, from which human care is appreciated, warm and personalized.
\end{abstract}

Keywords: humanized-birth; nursing-services, perinatal-nursing; social-perception.

DOI: https://doi.org/10.15517/revenf.v0i35.33666

${ }^{1}$ Date of receipt: March 1, 2018

Date of acceptance: June 18, 2018

2 Doctor in Nursing. Professor and Researcher at the Faculty of Nursing and Obstetrics of the Autonomous University of the State of México. Toluca, México. E-mail: gomezdanelia@usa.net

${ }^{3}$ Nursing degree of the Faculty of Nursing and Obstetrics of the Autonomous University of the State of México. Toluca, México. Email: Gabriela Téllez Rojas. E-mail: gabrielatellezrojas@hotmail.com

${ }^{4}$ Doctor of Health Sciences. Professor at the School of Nursing of Coimbra, Portugal. E-mail parreira@esenfc.pt

${ }^{5}$ Doctor in Nursing. Professor at the Federal University of Paraná. Curitiba-Paraná-Brazil. E-mail: aidamaris.peres@gmail.com 


\title{
Percepção social de usuários atendidos exclusivamente por enfermeiros no estágio perinatal ${ }^{1}$
}

\author{
Danelia Gómez Torres ${ }^{2}$; Gabriela Téllez Rojas ${ }^{3}$; Pedro Miguel Santos Dinis Pareira ${ }^{4}$; Aida Maris Peres ${ }^{5}$
}

Instituição: Universidade Autônoma do Estado do México.

\section{RESUMO}

O artigo mostra a atenção exclusiva dos enfermeiros em uma maternidade, cujo objetivo foi analisar as representações sociais dos usuários sobre os cuidados prestados pelas enfermeiras obstétricas na instituição. Trata-se de uma pesquisa com abordagem qualitativa, descritiva; dirigido pela teoria das representações sociais de Moscovici. Participaram 34 mulheres atendidas na maternidade. A análise dos dados é realizada através de um quadro interpretativo, selecionando as ideias destacadas por meio da técnica cromática; em seguida, os dados empíricos confrontados com a teoria são discutidos. Os usuários indicaram que o atendimento recebido fazia parte de um quadro de humanização, responsabilidade e confiança, que possibilita atender a um parto de acordo com seus costumes, o que, baseado na teoria, reflete a autonomia profissional. Conclui-se que o modelo assistencial mostra que o cuidado de enfermagem é baseado no conhecimento, experiência.

Palavras-chave: enfermagem-perinatal; parto humanizado; serviços de enfermagem; percepção social.

DOI: https://doi.org/10.15517/revenf.v0i35.33666

${ }^{1}$ Data de recepção: 1 de março de 2018

2 Doutora em Enfermagem. Professor e Pesquisador da Faculdade de Enfermagem e Obstetrícia da Universidade Autônoma do Estado do México. Toluca, México. Correio eletrônico: gomezdanelia@usa.net

${ }^{3}$ Licenciatura em Enfermagem pela Faculdade de Enfermagem e Obstetrícia da Universidade Autônoma do Estado do México. Toluca, México. Correio eletrônico: gabrielatellezrojas@hotmail.com

${ }^{4}$ Doutor em Ciências da Saúde pela Escola Superior de Enfermagem de Coimbra, Portugal. Correio eletrônico: parreira@esenfc.pt

${ }^{5}$ Doutora em Enfermagem. Professor da Universidade Federal do Paraná. Curitiba-Paraná-Brasil. Correio eletrônico:

aidamaris.peres@gmail.com 


\section{INTRODUCCIÓN}

Hasta hoy, la maternidad es la plataforma que muestra el actuar de la enfermería obstétrica y perinatológica. Al respecto, el profesional en enfermería mantiene una actitud comprometida frente a la realidad actual en cuanto a su práctica diaria, al ofrecer un cuidado humano, cálido y sensible, que refleje su crecimiento personal y profesional, pues durante la etapa gestacional las usuarias son atendidas exclusivamente por tales profesionales, lo cual proyecta un impacto transformador de la profesión. Así, la enfermería acelera su desarrollo como actividad científica dentro de las profesiones, al presentarse como un ejercicio de mayor competencia científico-técnica, ejercer un liderazgo en sus funciones a partir de sustento científico, al proyectar en su práctica laboral un nivel de actuación con mayor autonomía en los diferentes espacios sociales, de manera especial en las instituciones de salud. De igual manera, considerando que las unidades de cuidado obstétrico son parte fundamental del modelo de atención en salud, y forman parte de las estrategias de cualquier maternidad para proveer la atención de más alta calidad, debe contar con la infraestructura y personal necesarios para satisfacer la demanda de cuidados, respecto de la población demandante ${ }^{1}$.

La maternidad inicia sus servicios con base en las diferentes políticas de salud de entidades públicas; conociendo las necesidades de sus habitantes, los profesionales de enfermería aprovechan de forma oportuna la infraestructura de un hospital ya deshabilitado, para establecer un modelo de atención de enfermería innovador, dirigido a las mujeres de la zona, en su mayoría indígenas. De esta manera, la enfermería enfrenta los retos existentes en el sector salud, y los asume para apoyar al logro del Quinto objetivo de desarrollo del milenio establecido por la Organización Mundial de la Salud (OMS) -mejorar la salud materna- el cual pugna por reducir en tres cuartas partes la mortalidad materna, un desafío por cumplir y un indicador seleccionado para evaluar el progreso en tal reducción al ofrecer atención completa y con personal calificado para atender el parto ${ }^{2}$, y mejorar las condiciones de las mujeres durante la reproducción.

Al ser los servicios de la maternidad proporcionados por enfermeras obstetras y perinatales, se ofrece una atención más cercana y personalizada a la mujer embarazada, desde las primeras consultas prenatales, aspecto que facilita un proceso de vinculación emocional usuarias-enfermeras ${ }^{3}$, a través de un plan de atención que incluye diferentes intervenciones de enfermería, que enfatiza las educativas, para el adecuado control prenatal que requieren las mujeres con diagnóstico de gestación sin riesgo que inicia desde que se determina el embarazo, durante este, el nacimiento, puerperio y hasta los dos años de vida del infante.

Los servicios que ofrece la maternidad, a través de las enfermeras en el área de consulta externa, incluyen vigilancia prenatal, considerándolos como un aspecto que refleja los modelos de salud, ya que en ellos se lleva a cabo la detección oportuna de los factores de riesgo maternos y fetales, si se considera que 30\% de la mortalidad perinatal acontece durante el último trimestre del embarazo y otro $30 \%$ en las primeras horas del nacimiento ${ }^{4}$. Igualmente, durante el puerperio, la institución ofrece clínica de lactancia materna, planificación familiar, vigilancia del crecimiento y desarrollo del infante, estimulación temprana, psicoprofilaxis perinatal e intervenciones preventivas ${ }^{3}$.

La maternidad cuenta con sala de trabajo de parto, sala de nacimiento con instalaciones para cuidados perinatales, personal y equipo específicos para la atención integral y un ambiente que permite la vinculación entre usuaria y enfermera, lo que propicia el parto humanizado, considerando un escenario con modelo único. 


\section{Revista Electrónica Enfermeria Actual en costa Rica}

Esta maternidad se estableció con el objetivo de ofrecer cuidados durante el parto de acuerdo con los usos y costumbres de las comunidades indígenas, cuyas tradiciones se fundamentan en los cuidados culturales, ya que cada persona tiene su forma especial de concebir el proceso de vida y las formas de expulsar a su producto en posiciones diferentes, ya sea vertical-hincadas, en cuclillas o sentadas con la espalda apoyada ${ }^{3}$. De esta manera, la maternidad se establece como un nuevo modelo de atención, congruente con la cultura de la gestante, que influye en la generación de nuevas políticas de salud.

En lo concerniente a esta investigación, permitió conocer la trascendencia y aceptación de las usuarias de este servicio en cuanto a la imagen de la enfermera obstetra, de modo que se posibilite la expansión del modelo en otras latitudes.

Se instrumentó el proyecto de investigación, con el objetivo de analizar las representaciones sociales que tienen las usuarias sobre la atención que otorgan las enfermeras obstetras de la maternidad, fundamentado en la teoría de las representaciones sociales de Moscovici, considerada un eje conductor para el análisis y discusión de los resultados, la cual posibilita obtener la representación social que tiene el grupo de mujeres embarazadas de la que se obtuvo el objeto de estudio: ¿qué significa para la paciente la forma en que fue atendida en la institución? A partir de lo anterior, se relata las experiencias de las usuarias, en relación con el actuar de las enfermeras en su realidad y en un ambiente natural.

Tales estudios integran la difusión de los saberes, la relación entre pensamiento y comunicación y el origen del sentido común ${ }^{5}$, ya que una representación social se forma a través de pensamientos cotidianos cuyos contenidos se construyen y reconstruyen en hechos; en los que las creencias son el componente de formas diversas según las culturas y los grupos sociales ${ }^{6}$, tal como refiere Moscovici.

\section{MÉTODO}

La investigación tiene un enfoque metodológico cualitativo, apoyado en la percepción de las usuarias al ser atendidas en la institución; el estudio se llevó a cabo en un ambiente cotidiano para las participantes ${ }^{7}$. El trabajo es descriptivo, direccionado por la teoría de representaciones sociales de Moscovici ${ }^{8}$, lo que le convierte en el eje conductor del análisis y discusión de la intervención del personal de enfermería durante el proceso reproductivo.

Los actores sociales fueron 34 pacientes seleccionadas por haber sido atendidas y captadas desde el inicio de su embarazo en la maternidad. Su inclusión se debe a que participaron en el curso de educación para reproducción (psicoprofilaxis obstétrica), aunque se excluyó a aquellas que no firmaron el consentimiento informado y que solo llegaron a atender su parto a la maternidad.

El escenario de la investigación fue la maternidad localizada en el Estado de México, atendida exclusivamente por 36 enfermeras perinatólogas u obstétricas, particularidad que la distingue, ya que tiene como política ofrecer atención exclusiva por personal del mismo género, así como educación para la reproducción y servicios que cubren todo el proceso reproductivo. 


\section{Revista Electrónica Enfermeria Actual en costa Rica}

Las entrevistas fueron desarrolladas en un espacio físico situado en el área de consulta externa; para realizarlas se solicitó autorización a la Jurisdicción Sanitaria, así como a la Coordinación Normativa de Enseñanza: una vez aprobada la solicitud, se asignó el lugar para llevar a cabo la investigación.

Se formuló las preguntas conductoras, desprendidas de los objetivos del proyecto para así construir el instrumento, el cual fue una guía de entrevista que constó de 12 preguntas que evidencian los elementos del campo de las representaciones sociales, con el fin de investigar lo humano sin sacrificar su naturaleza. Las preguntas fueron abiertas e incluyeron los aspectos sociodemográficos de las entrevistadas, con el fin de obtener el conocimiento social del grupo por abordar y validar el instrumento en cuanto a la comprensión del lenguaje, el cual se aplicó a tres usuarias: posteriormente se efectuó los ajustes necesarios.

En cuanto a las entrevistas, se indicó que serían grabadas. El primer paso fue la presentación de la investigadora, posteriormente se invitó a las usuarias a participar, se les explicó el objetivo de la investigación, se leyó el consentimiento informado y se solicitó su autorización mediante su firma.

Las entrevistas fueron grabadas y transcritas en su totalidad: se leyó y releyó para identificar lo más relevante, además se extrajo las ideas más importantes (marcadas con colores), para codificarlas y jerarquizar la información de acuerdo con el referencial teórico, para una categorización posterior -por criterio semántico-, que fuese recurrente en aparición o relevancia implícita de lo que podía significar algo para el objetivo analítico seleccionado ${ }^{9}$ así establecer las categorías y subcategorías. El análisis de los datos fue mediante un marco interpretativo de contenido, para responder a las preguntas conductoras y a los objetivos planteados, así como para comprender el modo en el que las usuarias perciben el actuar de enfermería, desde la perspectiva de las representaciones sociales.

Se discutió los resultados, siguiendo el método de discusión científico; primero se conceptualizó la categoría y posteriormente se presentó el dato empírico para poder confrontarlo con el referente teórico, este fundamento posibilita plasmar el razonamiento lógico, producto del proceso de un análisis intelectual, discusión que fue apoyada en el referencial teórico postulado por Moscovici. En cuanto a la teoría, es una modalidad particular de conocimiento que contribuye a elaborar los comportamientos y la comunicación entre los individuos ${ }^{10}$ respecto de la percepción que las usuarias poseían del servicio obtenido, para lograr una en función de la diversidad de estímulos recibidos durante su estancia en la maternidad.

En cuanto a las limitaciones, se destaca que, al ser un hospital de reciente creación, aún no cuenta con un comité de ética de la investigación, por tanto, se debe anclar al que la jurisdicción sanitaria instale para tal efecto.

\section{CONSIDERACIONES ÉTICAS}

Para cumplir con los aspectos éticos de la investigación, en cuanto al anonimato y el sigilo de los participantes en la investigación, se utiliza un código para cada entrevistada, de modo que se cumpla con la Ley General de Salud en Materia de Investigación para la Salud, Título Segundo, Capítulo I, Artículo 14 fracción VIII, el cual señala que "se llevará a cabo cuando se tenga la autorización del titular de la institución de salud [...]" autorizó la investigación mediante oficio N. ${ }^{\circ} 217 \mathrm{~B} 200012 / 3269$ de fecha 2 de octubre de 2014. 


\section{Revista Electrónica Enfermeria Actual en costa Rica}

\section{RESULTADOS}

Respecto de la dimensión de las representaciones sociales, los aspectos sociodemográficos de las participantes, dan una visión general de aquellas características de los actores sociales que participaron, cuyos resultados fueron edad predominante - entre los 21 y 35 años-; su escolaridad fluctuó de nivel medio a superior; la mayoría casadas; en relación con la gestación, 19 de las 34 eran primigestas.

Los resultados se presentan por categorías: percepción de la atención, significado de atención, atención por personal de otro género, humanización, responsabilidad.

Percepción de la atención. En la construcción de esta realidad, las pacientes señalan que la atención recibida de las enfermeras durante el parto fue como se detalla a continuación:

Yo les digo mi experiencia, ellas les tienen mucha paciencia (...) a uno como mujer le atienden, están al pendiente (E-13).

El trato es muy bueno, intentan que todo sea natural, son muy cálidas, muy cercanas (...) como si fuesen tu familia, la mamá o la hermana, se preocupan mucho por tu comodidad (E-12).

Me atendieron bien, como es la primera vez, uno entra ... así nerviosa con miedo, pero después se va dando cuenta de que la atienden bien. No estuve sola, te están preguntando las licenciadas, si está uno bien, si te sientes cómoda (E-14).

Lo relatado muestra una atención óptima con elementos del entorno sociocultural, que integran un conjunto de intervenciones encaminado a satisfacer las necesidades de las usuarias.

Significado de la atención. La Organización Mundial de la Salud considera el cuidado materno como una prioridad de atención, además de una estrategia para optimizar los resultados del embarazo y así prevenir la mortalidad materna y perinatal ${ }^{12}$. Este aspecto es descrito por las usuarias de esta forma:

Fue mi primera experiencia que tuve y lo que significó para mi la atención que me dieron las enfermeras fue como si estuviera en mi casa, con personas de confianza (E-27).

La confianza es uno de los significados que son parte de la representación al ser las mujeres atendidas en el parto, por una profesional de enfermería, aspecto manifestado así:

Con más confianza, porque son mujeres, pues con los hombres me da temor (E-8).

Me agradó mucho que fueran en primer lugar mujeres, porque, como tal, entre mujeres nos entendemos, nos sentimos hasta con más confianza de decir lo que sentimos (E-5).

La confianza que inspiró la enfermera a la paciente durante su atención del parto, generó el marco para expresar y compartir las experiencias vividas, las cuales fueron señaladas de esta manera:

Lo ves en las citas, como te van atendiendo ya vas creando esa confianza relajada (E-9). 
Por lo regular todos los que atienden los partos son médicos y vi que eran enfermeras y dije: vamos a probar. Es un buen servicio y que haya sido una mujer, pues muy bien, porque hubo más confianza, es como más comodidad, fue algo que tuvo importancia en mí (E-21).

Me dieron toda la confianza de poder sentirme libre, de decir cómo me siento, porque a veces no sabes ni cómo expresar lo que sientes, pues tú piensas qué van a decir de ti. No, esta vez aquí fue diferente. Las enfermeras me dieron toda la confianza y me dijeron háblanos, grítanos o toca el timbre, como tú quieras. Me sentí muy segura y tranquila durante el parto (E-31).

Atención por personal de otro género. En este aspecto las usuarias experimentan cierta inquietud respecto de ser atendidas por personal masculino, lo cual las impacta de manera negativa:

Estuve en el hospital, me atendió un médico y me hizo sentir muy incómoda porque era hombre, entonces dije ¿cómo, un médico me va a estar revisando?, y cuando llegué aquí en la maternidad, me revisaron las licenciadas, fue muy diferente, como una experiencia más agradable, con más confianza (E-13).

Me sentí cómoda, pues porque fueron puras mujeres, porque igual a mí ni me gusta eso de los hombres. Hace cuatro años que tuve a mi hija me atendieron en el hospital y, pues, me sentí incómoda porque fueron hombres los que me atendieron (E-31).

La humanización: como en toda práctica, debe favorecer al trinomio madre-hijo-padre, en este sentido, la enfermería es una de las profesiones que tiene mayor acercamiento con el paciente y su familia durante la intervención y esa calidez se refleja en los siguientes comentarios de las usuarias:

Bueno, a mí en lo personal me agradó muchísimo, ya que siempre que veníamos a consulta o alguna revisión, fueron muy amables, yo siento que son muy humanas, se ponen en tu lugar (E-5).

Ya había pasado esa experiencia en el otro hospital, me mandaron a caminar, pero no salí y quede ahí adentro, cada que me daban los dolores, me acercaba a los médicos, a veces no me hacían caso, a veces sí, pero aqui fue muy diferente, siempre están pendientes de uno, te dan agua, si quieres bañarte, te puedes bañar, son muy humanas (E-7).

Que no pierdan el sentido humano que las caracteriza, que sigan conservando su humanidad que muestran con nosotras, es lo que nos beneficia para sentirnos en conexión con ellas (E-5).

Responsabilidad: la responsabilidad en la atención de las gestantes, se proyecta por un juicio de valor que las enfermeras poseen, así confieren seguridad en su actuar, manifestado por las usuarias de la siguiente manera:

Me trataron muy bien, me gustó mucho porque en cuanto llegué me preguntaron cómo me sentía. Las enfermeras me revisaron, nunca me dijeron "espérese, luego la atendemos", estuvieron conmigo todo el tiempo, nunca me dejaron sola. Ellas son responsables con lo que hacen (E-7).

Aquí llegué cuando tenía dolores, todavía no eran muy fuertes y como venimos de lejos ya no me quisieron regresar adonde yo vivo y me dieron albergue. Me dormí, ya como a las tres de la mañana me empezaron a dar los dolores más fuertes, las enfermeras fueron responsables conmigo (E-8). 


\section{Revista Electrónica Enfermeria Actual en costa Rica}

\section{www.revenf.ucr.ac.cr}

Pues me sentí con mucha confianza, más que nada agradecida con ellas, su responsabilidad con nosotras es muy diferente y muy bonito, como una experiencia más agradable, con más confianza más que nada (E-13).

\section{DISCUSIÓN}

Durante las últimas décadas del siglo XX se empezó a cuestionar los modelos medicalizados de atención al nacimiento, tema que generó un intenso debate internacional y que inició una nueva tendencia en relación con la forma en que debía atenderse el nacimiento, cuando el embarazo y el parto se desarrollaban con normalidad ${ }^{13}$. La atención en el parto se ha transformado en un acontecimiento fundamentado en un modelo hegemónico, que deja de lado la atención con elementos de un entorno sociocultural, mientras que en la maternidad se ha rescatado el modelo en el que las gestantes reciben atención sanitaria óptima, basada en un conjunto de servicios y diagnósticos terapéuticos.

En este caso, la percepción de la atención del parto es uno de los aspectos que las usuarias proyectan según su representación social, , que permite la construcción de una realidad común en un conjunto social. Por eso, es necesario la reflexión, discusión y ponderación, considerando el conocimiento específico, los valores, los principios éticos y legales, así como las normas o reglas de conducta agregadas ${ }^{14}$, ya que todo se ordena a partir de lo figurativo que, de algún modo, condensa todas las imágenes, todas las nociones o los juicios que un grupo o una sociedad genera a lo largo del tiempo ${ }^{15}$. Lo anterior se manifiesta como seguridad, comodidad y bienestar, inherentes al rol de la enfermería obstétrica, construyendo así una realidad común respecto de las experiencias de vida $^{16}$, las cuales se manifiestan en el actuar cálido y el buen trato que brindan las enfermeras obstetras durante la atención ofrecida.

De igual manera, un parto debe desarrollarse en las mejores condiciones de intimidad, respeto, comodidad y libertad, así la madre dará a luz en un ambiente óptimo, a lo que se suma un espacio físico ${ }^{11}$ que facilite el reconocimiento de ambientes sociales armónicos; las expresiones relacionadas con la confianza, según los hallazgos, reproducen percepciones retenidas por el recuerdo, que se expresa en la realidad ${ }^{15}$, en las cuales la gestante y la enfermera comparten diversas emociones, a raíz de la experiencia, fortalecidas por una atención de confianza, en la que prevalece un entorno de familiaridad, donde la gestante tiene la libertad de desenvolverse.

Respecto de los servicios de la maternidad, la percepción de las usuarias se apoya en sus vivencias, considerando que las representaciones sociales se manifiestan con palabras, sentimientos y conductas; por tanto, deben ser analizadas a partir de la comprensión de estructuras y comportamientos sociales ${ }^{15}$. De forma congruente, la confianza fue el valor intangible que influyó en situaciones de crisis ${ }^{11}$, entre quien solicita la atención obstétrica y quien la propicia, lo cual genera un comportamiento de intimidad entre pares en el que la atención de parto brindada por una mujer les inspira confianza.

En torno a la confianza, sin duda, se constituye en la clave de toda acción, ya que implica involucrar al "otro" en todo proceso humano, por tanto, se trata de un concepto cuya interpretación es necesariamente interdisciplinar, ya que requiere conocer todo sobre la naturaleza humana ${ }^{16}$. También es el reflejo de la atención a la paciente, descrita como una relación de ayuda que permite conocer y a su vez tener autocontrol. La intervención de la enfermera durante la etapa gestacional, propició un clima de seguridad en el que las usuarias tienen libertad de 


\section{Revista Electrónica Enfermeria Actual en costa Rica}

\section{www.revenf.ucr.ac.cr}

expresar sus sentimientos tanto positivos como negativos, en relación con la etapa que están viviendo, así como expresar inquietudes y temores, propios de toda mujer próxima a ser madre.

Dado lo anterior, los vínculos de confianza entre paciente y enfermera, fundados en las relaciones intersubjetivas construidas durante la atención, cambiaron radicalmente, respecto a las que se establecen entre sí, el lugar que ocupan y el sentido que tienen en la asistencia y cuidado por lo que se refiere a lo tradicional ${ }^{19}$. En contraparte, la incomodidad como alteración del bienestar de la paciente, se presenta cuando la atención es brindada por hombres, ya que la intervención obstétrica en el modelo institucionalizado provoca incomodidad, entre lo natural y lo cultural, hasta generar la pérdida de la autonomía y la capacidad de decidir, así lo reflejan las declaraciones de las participantes, cuando relatan que la tención otorgada por un médico les genera incomodidad, debido a la poca comunicación que existe entre el profesional y la usuaria.

De acuerdo con las acciones en torno a la humanización, se infiere que al momento que la enfermera desarrolla una función esencial, participa y promueve un clima de humanización, en el cual se respeta la decisión de la mujer, especialmente durante el parto, de modo que se cumpla con la responsabilidad ética y de protección al derecho a la vida de los seres humanos, desde la concepción hasta la muerte, al respetar su dignidad, integridad genética, física, espiritual y psíquica ${ }^{17}$.

Las representaciones sociales como modo individual del conocimiento de una sociedad permiten intercambiar experiencias, conocimientos y vivencias ${ }^{20}$, propuesta que es congruente con el significado de las usuarias acerca de la intervención de la enfermera; además, sugieren que conserven el humanismo, distintivo del cuidado que brinda la enfermera en la atención del trinomio madre-recién nacido-padre.

La responsabilidad del desarrollo profesional de las enfermeras se debe dimensionar de acuerdo con parámetros de obligación ${ }^{14}$; por ende, es fundamental comprender el proceso de trabajo de enfermería, como una práctica social destinada a un propósito, que debe ser utilizado como instrumento para atender un objetivo específico ${ }^{21}$ : la enfermera responde por sus actos frente a los demás, y manifiesta que la buena práctica de la profesión consiste en la aceptación de nuevos principios y valores del ejercicio.

En los hallazgos de la investigación se proyectan las imágenes que la sociedad tiene respecto de las enfermeras. Las ideas en las representaciones sociales forman juicios de valor que los individuos dotados por una voluntad poseen; por lo tanto, las concepciones sobre lo real tienen una dinámica propia de indudable importancia ${ }^{22}$, al igual que las necesidades de las gestantes las cuales, según sus juicios de valor, implican la responsabilidad de las enfermeras al satisfacerlas con humanismo y seguridad. De acuerdo con los hallazgos, el significado de la responsabilidad, en perspectiva de las representaciones sociales, se convierte en un juicio de valor proyectado de parte de la gestante a la enfermera, aspecto significativo para ellas.

\section{CONCLUSIONES}

Mediante el análisis crítico y reflexivo, la investigación posibilitó determinar la manera en que las enfermeras asumen una perspectiva humanizada, al atender a mujeres en etapa perinatal. Además, la representación social más destacada por parte de la paciente de maternidad, una vez analizados los hallazgos, fue de responsabilidad y confianza, la cual pasó a tener un significado o símbolos, que las conduce a actuar en una vertiente cognoscitiva, 


\section{Revista Electrónica Enfermeria Actual en costa Rica}

afectiva y técnica, con componentes integradores -enfermero, proceso e institución- tal como lo establece el principio de las representaciones sociales.

Los resultados analizados nos permiten indicar que la actuación de la enfermera en una unidad hospitalaria de esta naturaleza, contribuye a la práctica obstétrica, para alcanzar los objetivos establecidos a nivel internacional en materia de salud reproductiva, con los que México se ha comprometido. Así, en el modelo de atención que ofrecen las enfermeras, se vislumbra como prototipo de atención que posibilita establecerse como estrategia de centros de atención basados en un proceso formativo que favorezca la reproducción.

\section{CONFLICTOS DE INTERÉS}

Cabe señalar que esta investigación no presenta conflictos de interés económicos ni de otra naturaleza.

\section{REFERENCIAS BIBLIOGRÁFICAS}

1. Fajardo QJC, Cruz SM, Mora Y, Torres LLM. Validación facial de la escala Nursing Activities Score en tres unidades de cuidado intensivo en Bogotá, Colombia. Rev. Global. 2017;102-115. http://dx.doi.org/10.6018/eglobal.16.1.261091

2. Aranzabal AM, Canadell VD. Salud en el mundo y objetivos del milenio. Rev Pediatr Aten Primaria. 2014; 16(1):199-203.

3. Instituto de Salud del Estado de México (2013). La maternidad de Atlacomulco: modelo de atención de la mujer y del menor de 2 años basado en atención primaria de la salud, México: Kaleidoscopio.

4. Rodríguez-Reyes ER, Márquez-González H, Castañeda-Casale G. Mortalidad perinatal en un hospital de segundo nivel de atención. Rev. Med IMSS. 2010; 48(3): 237-242.

5. Ribeiro L P, Antunes-Rocha MI. (2016) Social Representation Theory: history, approaches, methods and perspectives Rev. Social Representation Theory: history, approaches, methods and perspectives, Rev. Psicologia \& Sociedade. 2016; 28(2), 407- 409.

http://dx.doi.org/10.1590/1807-03102016v28n2p407

6. Mora, Martín. La teoría de las representaciones sociales de Serge Moscovici. Rev. Athenea Digital. 2002.

7. López SE. Crisis en la enseñanza de la metodología cualitativa. Andamios. Revista de Investigación Social. 2016; (13) 109-127.

8. Moscovici S. (2012) Representações sociais, investigações em psicologia social. Brasil: Vóces

9. Zerbetto, SR, Martins de Souza Gonçalves, A, Santile, N, Frari Galera, SA, Acorinte, AC, Giovannetti, G. (2017) Religiosidade e espiritualidade: mecanismos de influência positiva sobre a vida e tratamento do alcoolista. Escola Anna Nery Revista de Enfermagem. 21(1):1-8. 


\section{Revista Electrónica Enfermeria Actual en costa Rica}

10. Villamañan AM. (2016) Lo comunitario en las representaciones sociales de la violencia. Psicologia \& Sociedade; 28(3):494-504.

11. Secretaría de Salud (2012). Ley General de Salud. [En línea]. México.

12. Hernández AM; Vásquez ML. (2015). El cuidado de enfermería comprometido: Motor en la satisfacción de la gestante durante el control prenatal. Rev Univ. Salud. 17(1):80-96.

13. Goberna TJ. (2018) Bioética de la relación asistencial: El modelo deliberativo como propuesta frente a la falta de respeto, el maltrato y la violencia obstétrica en atención al parto. Dilemata, Núm. 26. 53-62.

14. Silva TN, Freire MEM, Vasconcelos MF, Silva Jr SV, Silva WJC, Araújo PS. (2018) Deontological aspects of the nursing profession: understanding the code of ethics. Rev Bras Enferm; 71(1):3-10. DOI: http://dx.doi.org/10.1590/0034-7167-2016-0565

15. Moscovici S, (2009) Os ciganos entre perseguição e emancipação. Sociedade e Estado 2(4) 653-678.

16. Carrascal G, Montenegro RJD. (2015) Higiene: cuidado básico que promueve la comodidad en pacientes críticos. Rev. Enf Global. 14(3)340-350.

17. Parada RDA, Olivares PE. (2015) Humanización del cuidado durante el encuentro madre-recién nacido: una responsabilidad ética del equipo de salud. Rev. Col Bioética, 10(1)134-147.

18. García ES. (2016) La confianza clave de la acción científica, económica y empresarial la dimensión económico-social de la acción humana. Madrid España: Instituto de Dirección y Organización de Empresas. 254-5.

19. Azeredo YN, Schraiber LB. (2016) El poder médico y la crisis de los vínculos de confianza en la medicina contemporánea. Salud colectiva. 12, (1). https://doi.org/10.18294/sc.2016.864

20. Perozo FML. (2014) Representaciones sociales en consumidores de drogas. Tesis Psicológica, 9(1)146-155.

21. Michelan VCA, Spiri WC. (2018) Perception of nursing workers humanization under intensive therapy. Rev Bras Enferm [Internet];71(2):372-8. DOI: http://dx.doi.org/10.1590/0034-7167-2016-0485

22. Sánchez VMJ, Blas LH, Ortiz OV. (2015) Bioética y salud mental: reflexiones sobre la afección y la responsabilidad en la relación profesional-paciente. Rev. Latinoamericana de Bioética. 15(2) 96-107. 